\title{
Enfoques y Prácticas de Extensión Rural Públicas en el Noreste Argentino ${ }^{1,2}$
}

\author{
Fernando Pablo Landini ${ }^{3}$
}

Resumen: Los enfoques y prácticas de extensión se han transformado notablemente en las últimas décadas. A la vez, numerosos autores han señalado la persistencia de enfoques difusionistas tanto en las instituciones como en las prácticas de los extensionistas. En esta investigación se analizan las prácticas de extensión implementadas en el noreste argentino, se las compara con propuestas institucionales y académicas actuales y se extraen aprendizajes. Para esto se realizaron 40 entrevistas a extensionistas que trabajan en el ámbito público en las provincias de Chaco, Corrientes, Formosa y Misiones. El estudio muestra que los entrevistados orientan sus prácticas a la mejora productiva y al fortalecimiento de las organizaciones de productores, valoran organizar su trabajo según la demanda y priorizan abordajes grupales. Las tres áreas de acción más mencionadas son la asistencia técnica, la articulación interinstitucional y el trabajo grupal. En términos generales, se observa una práctica consistente con la propuesta del Instituto Nacional de Tecnología Agropecuaria y con buena parte de los lineamientos de la literatura especializada, aunque existen dudas por el valor real dado al trabajo grupal, la articulación interinstitucional y la participación, y preocupación por la falta de interés en relación a temas como género y evaluación de proyectos.

Palabras-clave: Extensión agraria; Asistencia técnica; Participación; Enfoque territorial; Trabajo grupal.

Abstract: Extension approaches and practices have changed enormously during the last decades. At the same time, several authors have pointed out the persistence of traditional, diffusionist approaches in extensionists' practices as well as in extension institutions. In this research, extension practices implemented in the Argentine Northeastern region are analyzed and compared with institutional and current academic proposals, aiming to draw useful recommendations. In order to do so, 40 interviews with rural extensionists who work

1. Data de submissão: 14 de julho de 2015. Data de aceite: 15 de janeiro de 2016.

2. Este artigo deriva de um projeto de pesquisa financiado conjuntamente pela Agência Nacional de Promoção Científica e Tecnológica (ANPCyT) do Ministério da Ciência, Tecnologia e Inovação (MINCYT) e da Universidade de Cuenca del Plata (Argentina). O projeto é de código PICT 0192-2011.

3. Consejo Nacional de Investigaciones Científicas y Técnicas (Argentina), Universidad de la Cuenca del Plata (Posadas, Argentina), e Universidad de Morón (Buenos Aires, Argentina). E-mail: landini_fer@hotmail.com 
in the public system in the provinces of Chaco, Corrientes, Formosa and Misiones were conducted and analyzed. Results show that the interviewees aim their practices at the productive improvement and the strengthening of farmers' organizations, value implementing demand-driven interventions, and prioritize group approaches. In general terms, the extensionists' practices are consistent with the National Institute of Agricultural Technology's proposal and with most of the guidelines provided by the specialized literature. Nonetheless, doubts remain with regards to the real value given to group work, interinstitutional articulation and participation. Likewise, the lack of interest about gender issues and evaluation of extension projects are also cause of concern.

Key-words: Agricultural extension; Technical assistance; Participation; Territorial approach; Group work.

\section{Introducción}

Los enfoques y las prácticas de extensión rural (ER) se han transformado enormemente a lo largo de las últimas décadas a nivel mundial (LEEUWIS, 2004). A mediados del siglo pasado, hablar de ER significaba hablar de la transferencia o difusión de las tecnologías generadas en los centros de investigación a los productores del campo (ROGERS, 1962). Con el paso del tiempo este enfoque fue criticado, tanto por las dudosas premisas éticas en las que se sostenía (FREIRE, 1973) como por su limitado impacto en el trabajo con amplios sectores de productores, especialmente los agricultores de tipo familiar.

$\mathrm{Si}$ bien pueden encontrarse múltiples definiciones, actualmente tiende a considerarse a la ER como un conjunto de acciones orientadas a ofrecer información y servicios a agricultores y a otros actores rurales con el fin de desarrollar destrezas y fortalecer prácticas técnicas y organizativas, procurando mejorar la calidad de vida de los productores rurales (FORO GLOBAL PARA LOS SERVICIOS DE ASESORÍA RURAL [GFRAS], 2012). Siguiendo lineamientos propuestos por instituciones de extensión, organizaciones internacionales e investigadores, hoy la ER aparece como un proceso participativo, orientado a la demanda (ORTIZ et al., 2011; TRIGO et al., 2013), que se estructura a partir del intercambio horizontal de conocimientos $\mathrm{y}$ saberes entre productores y extensionistas (ORTIZ, 2009; PÉREZ y CLAVIJO, 2012), pero que a la vez requiere de la articulación dinámica y creativa entre diferentes actores e instituciones que actúan en un territorio o que forman parte de un mismo sistema de innovación agrícola (NEDERLOF et al., 2010; ACUNZO et al., 2014). En este contexto, la innovación deja de ser vista como el resultado de un proceso de transferencia de tecnologías predefinidas, pasando a ser concebida como el emergente de un proceso complejo y sistémico que involucra a diferentes actores (LEEUWIS, 2004; CHRISTOPLOS, 2010; SULAIMAN y DAVIS, 2012).

A nivel conceptual es posible establecer una tipología de enfoques de ER. Estos enfoques, si bien cada uno tiene una aparición histórica temporalmente delimitada, no deben ser pensados en términos de un conjunto de etapas en las cuales una supera a la otra, sino del surgimiento de la posibilidad de pensar de manera diferente, generalmente más amplia, los procesos de ER. Así, más que se superación de modelos, a nivel de terreno será más frecuente encontrar tensiones e hibridaciones entre ellos, más que enfoques puros, lo que ha sido argumentado en Argentina por diferentes autores (TORT, 2008; LANDINI, 2015a).

El primer modelo corresponde al enfoque clásico de transferencia o difusión jerárquica y lineal de tecnologías de investigadores y 
extensionistas a productores. Este enfoque, importado de los Estados Unidos por la amplia mayoría de los países latinoamericanos a mediados del siglo XX (DE LELIS et al., 2012), es descripto por Leeuwis (2004) como una intervención comunicativa orientada a "la persuasión de productores agropecuarios u otros grupos objetivo para que adopten paquetes tecnológicos específicos y/o acepten ciertas ideas o políticas" (p. 35). En contraposición a este modelo, en los años 70 comienza a hablarse en América Latina de un enfoque alternativo, el cual Freire (1973) denominó ER dialógica y que otros autores denominan ER crítica (TOMMASINO et al., 2006). En esta perspectiva, se reconocen los conocimientos y experiencias de los productores (SOUZA y GOMES, 2008), estableciéndose vínculos horizontales de diálogo y construcción conjunta de alternativas superadoras entre técnicos y productores (MACHADO et al., 2006; ZUIN et al., 2011). En Brasil, esta perspectiva se encuentra presente de manera clara en la Política Nacional de Assistência Técnica e Extensão Rural (PNATER) (MINISTÉRIO DO DESENVOLVIMENTO AGRÁRIO, 2004).

En este contexto cabe destacar que tanto el enfoque difusionista como el dialógico adoptan diferentes versiones. Por tanto, no resulta apropiado pensarlos en términos de enfoques únicos o monolíticos. Por ejemplo, dentro del difusionismo pueden encontrarse propuestas más centradas en un abordaje educativo, mientras que otras ponen el énfasis más en la transferencia directa de paquetes. Respecto del enfoque dialógico, debe señalarse que no todas las propuestas comparten la orientación a la transformación social presente en los trabajos de Freire, y que dentro del abordaje existen líneas que revalorizan las propuestas tecnológicas, pero no desde la transferencia lineal sino desde la adecuación o la co-construcción participativa.

Los modelos difusionista y dialógico de ER pueden considerarse como los enfoques clásicos de ER en América Latina. Ambos, a pesar de sus diferencias, ponen el foco en el vínculo entre técnicos y productores. En contraste, existen dos abordajes más actuales que cambian el nivel de análisis, haciendo énfasis en la articulación entre diferentes instituciones y actores sociales vinculados con los procesos de desarrollo rural, aún teniendo en cuenta que ambos incluyen enfoques similares pero que también admiten cierta diversidad a su interior. Así, por un lado, encontramos propuestas que piensan a la ER desde un enfoque territorial (ARDILA, 2010; RINGUELET, 2010), las cuales ponen el foco en la articulación de los actores locales con el fin de generar iniciativas de desarrollo endógenas que coordinen los recursos disponibles. El segundo abordaje, por su parte, centra su atención en el carácter sistémico de los procesos de innovación (LEEUWIS, 2004; LEEUWIS y AARTS, 2011). En este sentido, se destaca el rol de los ER como facilitadores de procesos articulación y de aprendizaje en el contexto de estos sistemas, de los que pueden forman parte productores, instituciones, consumidores, investigadores, comerciantes, cooperativas y cámaras empresarias, entre otros, no necesariamente articulados en torno a un mismo territorio.

No obstante, como se fue sugiriendo, contar con esta tipología no resuelve la cuestión de la diversidad de enfoques de ER existentes, ni a nivel conceptual ni a nivel práctico. De hecho, es posible encontrar abordajes tanto difusionistas como dialógicos articulados con propuestas territoriales o de sistemas de innovación (LANDINI et al., 2013; LANDINI, 2015a). A la vez, existen múltiples variables, como el carácter participativo de los enfoques, o el predominio de un abordaje técnico-productivo frente a uno más orientado a trabajar con el fortalecimiento grupal (o viceversa), que pueden agregarse caracterizando a los diferentes enfoques de ER mencionados anteriormente en la tipología. De cualquier manera, la tipología propuesta sí contribuye a aportar un orden a esta diversidad, lo que resultará de gran utilidad en este trabajo.

Por otra parte, la transformación histórica de los enfoques de ER a nivel académico y de organismos internacionales como la Organización de las Naciones Unidas para la 
Agricultura y la Alimentación (FAO) o GFRAS, no niega la persistencia de autores con enfoques tradicionales (véase por ejemplo MIRANDA et al., 2011; ADGABA et al., 2014; MANZANA et al., 2014; OLORUNFEMI et al., 2014). A la vez, estos cambios académicos tampoco implican una transformación en los lineamientos utilizados por las instituciones de ER para guiar sus prácticas, ya que en ellas pueden persistir, explícita o implícitamente, presupuestos difusionistas, tanto en sus objetivos institucionales como en sus estructuras de funcionamiento (véase SARAIVA y CALLOU, 2009). Por ejemplo, el caso de instituciones que argumentan la importancia de enfoques participativos pero cuyos proyectos se construyen a partir de una lógica vertical (LANDINI, 2013a). Finalmente, también es necesario reconocer la persistencia de extensionistas con enfoques difusionistas, como señalan diferentes autores (LANDINI, 2012; TURIJÁN et al., 2012; LANDINI y BIANQUI, 2014a; PAVÓN, 2014; LANDINI, 2015b; RENDÓN et al., 2015) los cuales se derivan con gran facilidad de la formación técnico-productiva que los profesionales de las ciencias agrarias suelen recibir en las universidades. De hecho, la persistencia de un enfoque difusionista en la ER pública brasileña ha sido identificada como un problema fundamental (SARAIVA y CALLOU, 2009; ALMEIDA et al., 2010; ZUIN et al., 2011; DE LELIS et al., 2012; LANDINI, 2015c), pese a las propuestas dialógicas, participativas y críticas derivadas de la PNATER.

Desde una perspectiva orientada al actor (LONG, 2007), la determinación de las prácticas de ER (es decir, los modos de acción concretos que implementan los extensionistas para alcanzar sus objetivos) puede ser pensada a partir del siguiente esquema, derivado de la propuesta conceptual desarrollada por Landini et al. (2014). En primer lugar, los extensionistas son portadores de una visión del mundo y de una concepción de ER a partir de la cual se proponen guiar su práctica. Ahora bien, como diferentes estudios han sugerido, las personas pueden tener diferentes concepciones, creencias o teorías sobre la práctica referidas a un mismo ámbito de la realidad (PEIXOTO y PEREIRA, 2013), que puedan activarse de manera diferencial dependiendo del contexto (LANDINI et al., 2013). Así, se parte de que los extensionistas son portadores de diferentes visiones de ER que guían sus prácticas, pudiendo existir diferencias tanto entre los extensionistas como al interior de su propia subjetividad e identidad, lo que coloca a este estudio en el nivel de articulación entre lo psicológico y la práctica social.

Por su parte, queda claro que las prácticas de ER no se encuentran determinadas únicamente por las concepciones de los extensionistas, sino que también se encuentran enmarcadas tanto por las condiciones de posibilidad como por las limitaciones derivadas de las estructuras institucionales dentro de las que trabajan, los entornos socio-institucionales con los que interactúan y las condiciones propias del ambiente material (clima, suelos etc.). En este contexto, la incidencia del cambio de las concepciones de extensión a nivel de la academia y de instituciones técnicas especializadas como la FAO podrá influir en las prácticas de los extensionistas a través de dos vías fundamentales: su formación (tanto a nivel de las universidades y los institutos técnicos, como en servicio) y la incidencia en las instituciones de extensión. Así, queda claro que conceptualmente será posible encontrar diferencias y contradicciones entre los enfoques de extensión priorizados a nivel académico, los marcos institucionales de las agencias de extensión, las concepciones de ER de los propios técnicos y las prácticas que realmente se implementan en los territorios. Al mismo tiempo, se reconoce que estos diferentes niveles de determinación identificados, incluyendo las concepciones de ER de los propios extensionistas, llevarán a la implementación de una importante diversidad de prácticas en diferentes instituciones y territorios. A partir de este marco conceptual, en este artículo se opta no proponer a priori una tipología de prácticas de ER, buscando abordar esta diversidad desde una perspectiva empírica e inductiva. 
En este contexto, localizados en el noreste argentino, caracterizado por un alto porcentaje de agricultura familiar, surgen diversas preguntas, que corresponden a los objetivos específicos de este trabajo: ¿Cómo se caracterizan las prácticas de ER pública que se llevan adelante en la región? ¿En qué enfoque o enfoques se sustentan? ¿Cómo se relacionan estas prácticas y enfoques con las propuestas académicas actuales para el trabajo de ER? ¿Qué aprendizajes pueden extraerse de este análisis para fortalecer el trabajo que se lleva adelante con los agricultores? Buscando responder a estas preguntas, a continuación se presentan los resultados de un trabajo de investigación.

\section{Metodología}

Sellevóadelante una investigación cualitativa, de carácter exploratorio-descriptivo. Se realizaron 40 entrevistas semi-estructuradas a extensionistas que trabajan en el noreste argentino en las dos principales instituciones públicas que brindan ER a nivelnacional: el Instituto Nacional de Tecnología Agropecuaria (INTA) y la actual Secretaría de Agricultura Familiar (SAF). La región noreste está compuesta por cuatro provincias: Chaco, Corrientes, Formosa y Misiones. Diez entrevistas fueron realizadas en cada una de ellas, cinco a extensionistas de cada institución. Teniendo en cuenta que a nivel nacional la mayor parte de los extensionistas son hombres (LANDINI, 2013b), se procuró entrevistar al menos una mujer de cada institución en cada provincia, para no perder la visión de las mujeres sobre los temas estudiados. En total, 29 entrevistados fueron varones y 11 mujeres. A la vez, 36 de ellos contaban con formación técnica (fundamentalmente ingenieros agrónomos y veterinarios), y 4 con formación en ciencias sociales.

Las entrevistas se organizaron a partir de ejes de indagación y no de preguntas fijas, como corresponde a las entrevistas semi-estructuradas. Los ejes utilizados fueron:

- Trabajo de ER realizado / descripción de las prácticas de ER.
- Problemas que surgen en el trabajo de ER y dificultades en el vínculo con los productores.

- Objetivos que se busca obtener en el trabajo de ER.

- Utilidad y valor de la transferencia de tecnologías en el trabajo de ER.

- Características y concepto de un 'buen extensionista'.

A partir de las respuestas a estas preguntas, el entrevistador repreguntó con el fin de obtener descripciones más claras y amplias de los temas abordados.

Las entrevistas fueron transcriptas $y$ analizadas con el apoyo del software Atlas Ti siguiendo los principios generales de la teoría fundamentada. En concreto, se procedió a una primera lectura de los textos para identificar todos los fragmentos con información relacionada con los objetivos. Seguidamente, por un procedimiento inductivo, se fueron identificando categorías o áreas temáticas para ordenar los distintos temas abordados por los entrevistados, las cuales fueron utilizadas para categorizar todo el material, en línea con las propuestas de Taylor y Bodgan (1994) para análisis cualitativo de entrevistas. En este proceso, tanto los temas que se repetían en las entrevistas como aquellos destacados por el marco conceptual utilizado sirvieron como guías para el análisis. En total, 64 categorías temáticas referidas a elementos de la práctica o de la concepción de ER de los entrevistados fueron construidas, muchas de ellas con muy baja presencia cuantitativa en las entrevistas. En el apartado 'Resultados y Discusión' se presentan subtítulos en los que se agruparon (por afinidad temática) las categorías de mayor relevancia cuantitativa, las cuales son analizadas e interpretadas. Al mismo tiempo, la información recolectada por medio de las entrevistas es complementada y enriquecida con documentación institucional disponible sobre el trabajo de ER que llevan adelante el INTA y la SAF.

Cuando resulta oportuno y posible a partir de la información disponible, se indica cuántos entrevistados mencionaron una categoría 
determinada. Por ejemplo, cuántos extensionistas señalaron como objetivo de su accionar la generación de mejoras tecnológicas en las chacras de los productores, o cuántos indicaron que solían abordar en su trabajo temáticas vinculadas con el uso del agua. Esta estrategia ofrece un criterio cuantitativo para comparar la presencia de ciertas visiones y perspectivas, algo que suele pasarse por alto en numerosas investigaciones cualitativas. No obstante, téngase presente que las cantidades y porcentajes no son el resultado de una encuesta o de preguntas directas respecto de tales cuestiones. Al contrario, se trata de la cuantificación de comentarios y referencias espontáneas realizadas por los extensionistas frente a las preguntas abiertas realizadas durante la investigación. Así, si bien estos números permiten analizar la relevancia comparativa de ciertas afirmaciones de los entrevistados, no resulta apropiado interpretarlos como resultados absolutos, ya que su contexto es el de una investigación cualitativa.

\section{Resultados y discusión}

\subsection{Marcos institucionales}

$\mathrm{Si}$ bien las condiciones materiales e institucionales no determinan las prácticas de los actores (LONG, 2007), en este caso los extensionistas, es indudable que éstas se encuentran constreñidas por las estructuras institucionales que las enmarcan (LANDINI et al., 2014), generalmente expresadas en los objetivos que proponen las instituciones de ER o la estructura de los programas o proyectos que implementan. Esto se evidencia en las palabras de un extensionista que señala que "dentro de INTA están categorizadas las formas de trabajar, desde lo que son los diferentes programas, [...] los ‘Minifundios' [...] va desde capacitación en lo organizacional [...] los 'Cambio Rural' que es más de comercialización, de gestión de la empresa, los 'ProFam' son más vinculados a la parte tecnológica".
En el año 2009 se crea la Subsecretaría de Agricultura Familiar, actual SAF, dentro del Ministerio de Agricultura, Ganadería y Pesca de la Nación, como continuación de lo que en ese entonces era el Programa Social Agropecuario (PSA). El PSA tenía líneas de funcionamiento y prioridades claras y estables en el tiempo. No obstante, con la creación de la Subsecretaría estos lineamientos se volvieron anacrónicos, sin que se construyeran nuevos: "no hay una continuidad muchas veces institucional. De acuerdo a la persona que está al frente [...] son las instrucciones". Es cierto que, informalmente, el Secretario Nacional de la SAF señala como ejes de trabajo estratégicos la soberanía alimentaria, la tierra, el agua y el hábitat en el contexto de la agricultura familiar. No obstante, queda claro que se trata de ejes genéricos, pero no de principios operativos. De esta manera, se observa una SAF con lineamientos institucionales cambiantes y frágiles, que dependen de la voluntad de autoridades circunstanciales y dela disponibilidad contextual de recursos para acciones.

Por su parte, el INTA es una institución con mucha más trayectoria, ya que fue creada en el año 1956 (TORT, 2008). Si bien trabaja con distintos perfiles de productores, en la región se destaca el trabajo con pequeños y medianos productores, incluyendo en casos puntuales comunidades aborígenes. En el año 2004 el INTA genera un plan estratégico institucional para el decenio 2005-2015 (INTA, 2004) en el cual se propone un enfoque territorial para los procesos de desarrollo rural, siempre apuntando a la articulación y concertación entre actores y a la promoción de sistemas locales de innovación y conocimiento. Hacia el año 2014 el INTA consolida esta política con la creación de los Proyectos Regionales con Enfoque Territorial (PRET), los cuales tienen por objetivo el abordaje de las problemáticas específicas de cada territorio a partir de la complementación de recursos (institucionales y extra-institucionales) y de acciones de ER e investigación.

A nivel operativo, la amplia mayoría de las acciones de extensión que lleva adelante la 
institución se articulan en torno al Programa Federal de Apoyo al Desarrollo Rural Sustentable (ProFeder), que cuenta con cuatro instrumentos diferenciados según el perfil de productor, todos ellos con enfoque participativo: el ProHuerta, destinado a población carenciada de los ámbitos rural y urbano; el Minifundio, destinado a productores minifundistas; el ProFam, orientado a pequeños productores familiares; y el Cambio Rural II, que trabaja con pequeños y medianos empresarios agropecuarios. Cada uno de estos instrumentos, además de contar con un público beneficiario propio, estructura las acciones de los extensionistas según los parámetros y prioridades de cada uno de ellos.

\subsection{Objetivos de la extensión rural propuestos por los entrevistados}

Los objetivos, es decir, el 'para qué', o el 'a dónde se quiere llegar' con el trabajo de ER, constituyen una dimensión que estructura el enfoque y la práctica misma de los extensionistas. Dos objetivos son mencionados por más del $50 \%$ de los entrevistados. En primer lugar, 23 extensionistas establecen como eje de su accionar la generación de mejoras tecnológicas y productivas en los predios de sus productores, lo que incluye tanto aumentar los rindes y la producción como implementar prácticas ambientalmente más amigables. Por ejemplo, un extensionista menciona la necesidad de generar un "aporte desde el punto de vista tecnológico, mejorar ciertas prácticas que hace el productor". $\mathrm{Si}$ bien esto no habla necesariamente de la implementación de un enfoque difusionista de ER, como se ha explicado en la introducción, sí evidencia el predominio o el énfasis técnicoproductivo de la mirada que tienen los propios extensionistas de su función, en contraste con la falta de referencias a la idea de gestión de procesos de innovación, postura más amplia para pensar los cambios tecnológicos (LEEUWIS, 2004).

Paralelamente, 22 entrevistados plantean como objetivo de su trabajo la mejora o el fortalecimiento de las organizaciones de productores o, en términos más generales, el desarrollo de autonomía y autogestión por parte de los beneficiarios. En esta línea, una entrevistada argumenta que "si no hay organización, es imposible llegar a un desarrollo". Así, la organización aparece en sí misma como una herramienta que permite que el productor "no dependa de que nosotros [los extensionistas] estemos para que las cosas se sigan logrando". En este sentido, la organización social de los productores aparece como un prerrequisito para su autonomía, enfoque que considera la actividad de ER no desde lo productivo sino más bien en clave de institucionalidad y de poder. Así, la organización (asociada a la obtención de personería jurídica), permite gestionar recursos del estado y negociar con actores más consolidados que forman parte de las cadenas productivas y de los sistemas de innovación agrícola.

En esta misma línea, 15 entrevistados (10 ya considerados entre los anteriores) señalan la importancia de reconocer y construir al productor como actor de su propio desarrollo, algo asociado con "lograr autogestión de las organizaciones [de productores] [pero también] con lograr más poder de los sectores más excluidos". Claro está, estas 15 afirmaciones pueden ser ubicadas dentro de un continuo que va desde una perspectiva de autogestión más organizacional a otra que subraya más el actorazgo político. De todas maneras, y en cualquier caso, queda claro que nos encontramos aquí frente a una dimensión diferente del trabajo de ER, en paralelo con el enfoque técnico-productivo.

Ahora bien, podría pensarse que estos dos abordajes, uno más relacionado con lo técnicoproductivo y otro con lo organizativo-político, corresponden a dos estrategias de intervención diferenciadas, incluso contrapuestas. No obstante, a nivel empírico lo que parece observarse es una articulación, no siempre bien lograda, entre ambas líneas de pensamiento, con diferencias según las orientaciones personales de los propios extensionistas. Como explica un entrevistado de la SAF: "lo que queremos llegar está un poco balanceado entre lo productivo y lo organizativo". 
Indudablemente, para superar muchos problemas vinculados con los productivo (como el acceso a maquinarias), resulta potencialmente valioso trabajar la articulación y organización de los productores.

Por otra parte, también se observan tres objetivos adicionales referidos al trabajo de ER que han sido mencionados por entre el 25 y el 50\% de los entrevistados. En concreto, 13 extensionistas sostienen que el aumento de los ingresos de los productores, generalmente asociado a una mejora de las condiciones de comercialización, constituye uno de los objetivos de su trabajo. En segundo lugar, 10 extensionistas también incluyen dentro de los objetivos de su trabajo el asegurar a los agricultores familiares el acceso a diferentes derechos relacionados con salud, educación y agua para consumo, entre otros. Finalmente, también 17 entrevistados plantean como objetivo "mejorar la calidad de vida del productor". En algunos casos, esta idea de aumentar la calidad de vida se explica en términos de aumento de los ingresos o de un mayor acceso a servicios públicos. No obstante, podría decirse que la mejora de la calidad de vida constituye un objetivo genérico que es retomado por los entrevistados para expresar el impacto positivo que se espera que tengan en los beneficiarios acciones orientadas a alcanzar todos los objetivos anteriores.

En resumen, se observa una interesante articulación entre objetivos técnico-productivos y organizativo-políticos, a lo que se suma el apoyo a la comercialización y el acceso a servicios públicos. Este esquema lleva a diferentes reflexiones. Primero, que el tradicional énfasis productivo de la ER, muchas veces asociado a un enfoque difusionista, pareciera incorporar progresivamente la dimensión social en la que se encuentran inmersas las prácticas productivas. Segundo, que esta dimensión social trae aparejada la toma de conciencia de las relaciones de poder que atraviesan los procesos de desarrollo, como suele destacarse en el contexto de la ER dialógica (TOMMASINO et al., 2006). Y tercero, la incorporación al trabajo de ER de la comercialización en tanto el eslabón faltante para articular la producción con la generación de ingresos.

\subsection{Ejes del enfoque de extensión rural}

Durante las entrevistas, los extensionistas hicieron referencia a diferentes elementos que dan forma a los enfoques de ER con los que trabajan. En primer lugar, 33 técnicos (es decir, el 82,5\% de la muestra) mencionaron la importancia de la demanda y de la participación de los productores como elementos del trabajo de ER, lo que va en línea con las recomendaciones de diferentes organismos e instituciones (INTA, 2004; QAMAR, 2011; TRIGO et al., 2013), incluyéndose también en este caso la Política Nacional de Assistência Técnica e Extensão Rural de Brasil (MINISTÉRIO DO DESENVOLVIMENTO AGRÁRIO, 2004). No obstante, analizando en detalle las entrevistas se observa un predominio de referencias a la importancia de partir de la demanda de los productores (29 casos), frente a sólo 14 que hablan de participación. Cuando se habla de 'demanda', se está haciendo referencia a generar acciones de ER a partir de los pedidos tanto de beneficiarios como de instituciones. Así, lo que la demanda configura es el contenido de lo que se hace, por ejemplo, los temas sobre los que se capacita o sobre los que se ofrece asistencia técnica. Por ejemplo: "las capacitaciones en general se dan según la demanda". En contraste, cuando se habla de 'participación', a lo que se hace referencia no es sólo a acordar el contenido de las acciones con los otros, sino a diseñar la estructura misma de las propuestas con ellos: consensuar proyectos, construir conjuntamente los objetivos o acordar la dinámica de las capacitaciones (MONTERO, 2004). En sus palabras, un entrevistado plantea el sentido de la participación: "el enfoque es el de tratar de hacer un diagnóstico con la gente para relevar cuáles son los problemas que ellos visualizan, y en función de ello ordenar todo un trabajo, como para ver de qué manera podemos empezar a dar en conjunto respuestas a los distintos problemas". Así, atendiendo 
a la diferente presencia cuantitativa de estas perspectivas en los discursos de los entrevistados, se observa que si bien la idea de 'participación' aparece como un valor destacado, la tendencia pareciera ser la de subsumir esta idea dentro de la simple 'respuesta a la demanda', lo que claramente limita su fuerza como principio organizador del trabajo de extensión.

Aquí resulta interesante señalar que la participación y la orientación a la demanda, en distintos niveles, aparecen como valores de los enfoques dialógico, territorial y de sistemas de innovación. A la vez, en un estudio realizado en Argentina también se observó que la valoración de la participación caracteriza a extensionistas con orientación tanto difusionista como dialógica (LANDINI, 2015d). De esta manera, si bien la orientación a la demanda y la valoración de la participación constituyen elementos fundamentales del abordaje de ER de los entrevistados, no resultan suficientes para vincular a los extensionistas con uno de los cuatro modelos centrales presentados anteriormente en la tipología de enfoques de ER.

Otro eje a partir del cual pueden diferenciarse las prácticas de ER refiere al énfasis grupal o individual del trabajo con productores. En este contexto, 28 entrevistados describieron trabajos con grupos o incluso mencionaron su preferencia por trabajar de esta manera, frente a sólo 5 que destacaron intervenciones de tipo individual, fundamentalmente referidos a asistencia técnica. Visto esto, podría pensarse que existe una tendencia al trabajo grupal o asociativo en contraposición a lo que sería una asistencia técnica individual. Sin embargo, 8 entrevistados señalan que no existe una contraposición entre ambas alternativas, dependiendo cada una de las circunstancias: "hay momentos en que es necesario juntar a todo el grupo u organización, y hay momentos en que puntualmente vamos a hacer visitas a integrantes de esas casas". Así, no parece correcto contraponer estas alternativas sino reconocer su potencial utilidad contextual.

Ahora bien, lo que resulta llamativo son las razones esgrimidas por los entrevistados para justificar la preferencia o el predominio de estrategias de trabajo grupal. En concreto, de los 14 extensionistas que dieron sus argumentos, algunos más de un argumento, 9 hablaron de falta de personal y de recursos operativos para hacer asistencia individual, y 2 de requisitos de las propias instituciones de extensión. En contraste, sólo 5 mencionaron potencialidades intrínsecas del formato grupal: 1 habló del valor de los grupos para generar aprendizajes horizontales entre los productores y 4 de la utilidad de los grupos o asociaciones como vía para fortalecer la capacidad de gestión y negociación del agricultor. Visto esto, resulta llamativo el escaso reconocimiento, en el contexto de la argumentación referida al porqué del predominio del trabajo grupal con productores, de las potencialidades que tiene al abordaje grupal, más cuando entre los objetivos del trabajo de ER se mencionó con frecuencia el fortalecimiento de los grupos de productores. Pareciera como si a nivel de objetivos genéricos fuera más fácil reconocer el valor intrínseco del trabajo colectivo, haciéndose más difícil cuando se lo piensa en términos de prácticas concretas, asociadas más a los esquemas de formación y a los conocimientos técnicos que poseen los propios extensionistas. A la vez, también resulta preocupante el escaso reconocimiento de la potencialidad de los grupos de productores como herramientas de aprendizaje horizontal, como se sigue de las propuestas actuales sobre aprendizaje social, señaladas particularmente en el contexto del abordaje de sistemas de innovación (LEEUWIS y PYBURN, 2002).

Por su parte, 21 extensionistas abordaron el tema del vínculocon los productores, mayormente desde la perspectiva de lo que consideran que debería ser y no necesariamente de lo que se hace, por lo que los resultados respecto de este punto deben ser interpretados en ese contexto. Dentro de los comentarios más frecuentes se destaca la idea de que el vínculo entre técnicos y productores tiene que ser horizontal, no porque ambos sean iguales, sino porque los dos tienen conocimientos y experiencias valiosas, lo que lleva a plantear el trabajo de extensión como un 
proceso de compartir e intercambiar saberes. Uno de los entrevistados lo expresa de esta manera: "valoro mucho el extensionista que no se siente más que el compañero campesino. No va con la idea de 'yo sé y vos no sabés', [...] yo creo que el conocimiento se genera de ese intercambio [...], poder ir experimentando y viendo". En este contexto, el extensionista, al partir de que el productor posee conocimientos y capacidades valiosas, asume que no tiene que partir de decir a los productores cómo tienen que hacer, sino del diálogo de saberes: "se trata de impulsar esos cambios en lo productivo, pero siempre desde el punto de vista del compartir los saberes, jamás de 'bueno, esto es así'”.

Analizando el vínculo que proponen los entrevistados entre técnicos y productores, se observa claramente una visión dialógica de la ER (FREIRE, 1973; SCHALLER, 2006), en la cual la extensión es comprendida no como un proceso de transferencia sino de comunicación entre sujetos diferentes pero con igual dignidad. A la vez, también se observa una mirada constructivista del conocimiento y de la innovación, en el sentido de que ambos surgen como resultado del trabajo conjunto de diferentes actores, en línea con las propuestas del abordaje de sistemas de innovación (LEEUWIS y AARTS, 2011). No obstante, el carácter romántico o excesivo que muchas veces tienen estas expresiones lleva a pensar en hasta qué punto se trata de discursos realmente incorporados a nivel práctico por los extensionistas, y no simples expresiones de nivel discursivo. Incluso, esta duda se hace mucho más fuerte cuando se encuentran casos en los cuales un mismo entrevistado parece adoptar posiciones contrarias en diferentes momentos de la entrevista, con afirmaciones más explícitas referidas a modelos centrados en el diálogo, y otras más sutiles o implícitas referidas a un enfoque difusionista. Si bien esta afirmación puede resultar extraña, va en la línea con un trabajo realizado en Paraguay en el cual se concluyó por medios estadísticos que en un extensionista pueden coexistir a nivel implícito postulados tanto dialógicos como difusionistas (LANDINI et al., 2013), los cuales pueden activarse o hacerse salientes en diferentes contextos materiales o de sentido.

Ya con menor número de menciones, también aparecen otras referencias que dan forma a los enfoques de ER. En las entrevistas, 11 extensionistas hicieron referencia al rol central que juega la dimensión ético-política en el contexto del trabajo de ER, generalmente asociada al modelo de sociedad y de desarrollo que subyace a las intervenciones técnicas. En este sentido, los extensionistas destacan la contraposición entre el modelo asociado a la producción de commodities, y el rol que juega la agricultura familiar en la seguridad alimentaria y la potencialidad de la ER para generar poder (incluso a nivel político) de los sectores campesinos. Esto puede observarse en la siguiente cita: "si esa adopción, propuesta, acompañamiento, [...] no va de la mano con lograr soberanía del territorio, con lograr autogestión de las organizaciones, con lograr más poder de los sectores más excluidos, no tiene sentido dedicarle tiempo". Como ya se señaló, esta toma de conciencia de la dimensión política y de poder asociada al trabajo de ER suele destacarse en el contexto de ciertas líneas de pensamiento al interior de la ER horizontal dialógica (TOMMASINO et al., 2006).

A la vez, 9 entrevistados también señalaron la importancia de adoptar un enfoque de ER orientado a la agroecología, algo tradicionalmente propiciado por la SAF y por el programa ProHuerta del INTA, y apoyado también en Brasil en el contexto de la Política Nacional de Assistência Técnica e Extensão Rural (MINISTÉRIO DO DESENVOLVIMENTO AGRÁRIO, 2004). No obstante, se observa que el enfoque agroecológico corresponde a una visión que se encuentra en disputa, ya que también hay extensionistas que critican el rechazo a los agroquímicos, abogando por un uso apropiado de estos productos, sin necesidad de que sean excluidos de la producción.

Por su parte, también se observan entrevistados que destacan la importancia del trabajo con jóvenes y con enfoque de género, 
siendo 10 los que hacen referencia a la primera cuestión y 6 a la segunda. Visto esto, resulta llamativa la escasa cantidad de entrevistados que destacan espontáneamente la importancia de la dimensión de género en el contexto del trabajo de ER, en contraste con la importancia dada al tema por diferentes autores e instituciones (ORTIZ, 2009; FERRO, 2014). De hecho, esto resulta aún más preocupante si se observa que de esos 6 entrevistados, 4 fueron mujeres, lo que implica que sólo 2 de los 33 entrevistados varones mencionaron el tema. Finalmente, 9 entrevistados (8 del INTA), hicieron referencia explícita a la implementación de un enfoque territorial, en línea con el abordaje territorial de la extensión y el desarrollo rural propuesto por el INTA, lo que muestra la importancia de los marcos institucionales en la estructuración de las prácticas y los enfoques de ER.

\section{4. Áreas y prácticas de intervención}

A nivel operativo, los extensionistas describen diferentes áreas y prácticas de intervención. En este contexto, 7 de los entrevistados destacan la importancia de adoptar estrategias de ER flexibles, que se vayan adecuando a las necesidades de los beneficiarios. Uno de ellos lo explica de esta manera: "depende de las situaciones digamos, no hay un esquema armado". Las acciones referidas al trabajo de ER pueden categorizarse en dos áreas diferenciadas: la gestión de proyectos y las áreas de intervención. A continuación se desarrolla cada una de ellas.

\subsubsection{Gestión de proyectos}

Gran parte de las prácticas de los extensionistas se enmarcan en proyectos diseñados para implementar acciones en relación a grupos o instituciones específicas, las cuales pueden ser muy variadas. Estas acciones pueden incluir desde la implementación de una feria ganadera o la reforestación en una microcuenca, hasta la compra de herramientas o insumos para un grupo de productores. Así, gran parte del trabajo de ER queda encuadrado, siguiendo los requerimientos del INTA y de la SAF, a partir del ciclo del proyecto: diagnóstico, diseño, ejecución, seguimiento y evaluación.

En concreto, 28 extensionistas mencionaron el diseño de proyectos, 19 la implementación de diagnósticos y de otras dinámicas participativas, 8 el seguimiento y 2 la evaluación. La siguiente cita de un extensionista lo ejemplifica: "hicimos un proyecto en uno de los grupos [...], yo hice todo el árbol de ideas, [...] las preguntas, cuáles son las debilidades, las fortalezas". Reflexionando sobre estos resultados, se observa un mayor énfasis en lo que es el diseño del proyecto que en su evaluación, lo que si bien no resulta llamativo, sí invita a prestar mayor atención a la fase final de los proyectos, para poder generar procesos de retroalimentación y aprendizaje que permitan mejorar las dinámicas de trabajo.

$\mathrm{Si}$ bien respecto de este punto pueden observarse algunas diferencias entre el INTA y la SAF, el diseño de proyectos se encuentra enmarcado en lo que es la búsqueda de recursos para implementar acciones específicas. Es decir, el diseño de un proyecto tiene como función fundamental entre los entrevistados la búsqueda de fondos, tanto en la propia institución como en otras. Esto puede observarse en las palabras de uno de los extensionistas: "gestionamos un proyecto, la municipalidad consiguió rápidamente una perforación con tanque de agua y nosotros a través de [el Ministerio de] Desarrollo Social vinculamos un proyecto de 96 mil pesos para comprar una rueda de agua y hacer funcionar en un arroyo". Así, en este contexto, también se toma conciencia que el trabajo de ER implica, como tarea necesaria, la búsqueda y la gestión de recursos para poder implementar acciones con los beneficiarios.

\subsection{2. Áreas de intervención}

Las áreas o temáticas de intervención en las que trabajan los extensionistas son múltiples. Para clasificarlas se trabajó inductivamente. Puede diferenciarse entre aquellas que son 
fundamentales (mencionadas por el $90 \%$ o más de los entrevistados), las que son destacadas (al ser referidas por entre el 50 y el 75\%) y las que son relevantes (cercanas al 20\%). Las áreas de intervención fundamentales de los extensionistas entrevistados son tres: la articulación interinstitucional, el trabajo grupal o asociativo, y la asistencia técnica. La articulación interinstitucional fue mencionada por 39 de los 40 entrevistados, es decir, el 97,5\% de la muestra, lo que va en línea con las propuestas de ER territorial del INTA. Una extensionista de esta institución lo explica: "la parte de articulación es fundamental, es muy importante, articulación con las instituciones que están en el territorio". Aquí, se observa que la idea de articulación interinstitucional refiere a la coordinación, más aún, a la sinergización de las acciones que implementan diferentes actores a nivel local, no pudiendo faltar las instituciones de extensión y desarrollo rural, los gobiernos locales (municipios), y las organizaciones de productores, además de otra multiplicidad de actores que puedan ser relevantes en diferentes contextos.

A nivel descriptivo, la mayor parte de los extensionistas explica la utilidad de la articulación interinstitucional en términos de no superponer acciones y, fundamentalmente, de poder articular recursos de diferentes fuentes para generar acciones de mayor impacto. Por ejemplo: "la parte de Infraestructura [del gobierno] quizás cede un lugar para la feria franca. O el traslado a veces, uno aporta un poco de gasoil, el móvil aporta el municipio [...] Trabajamos en lo que podemos y aporta cada uno lo que puede de su institución". En este sentido, se habla de alcanzar "eficiencia en el aprovechamiento de los recursos del estado". En contrapartida, pareciera quedar en un segundo plano lo que sería coordinación a nivel de políticas o de planes de desarrollo a nivel de los territorios entre las instituciones, lo que resulta mucho más complejo pero que refleja de manera más profunda la propuesta de enfoque territorial del desarrollo rural (SCHEJTMAN y BERDEGUÉ, 2004).
Por su parte, 37 entrevistados (el 92,5\% de la muestra) hicieron referencia a trabajos de asesoramiento o asistencia técnica a beneficiarios en el ámbito productivo. Un extensionista lo plantea de esta manera: "nuestro trabajo es asistirlo técnicamente [al productor], el tema de la capacitación, el seguimiento de las actividades planteadas". Como se señaló, dentro de esta categoría se incluyen diferentes actividades relacionadas con el asesoramiento productivo a los beneficiarios. Entre ellas sobresalen dos. La primera refiere a asesoramientos puntuales, generalmente a productores individuales en respuesta a problemas productivos concretos, siempre bajo demanda: "vos vas a ver a la familia que te viene a buscar porque [...] se le están muriendo las gallinas, te vienen y te piden que vayas". A esta práctica a veces se la denomina "asistencia técnica clásica". La segunda corresponde a lo que son capacitaciones, talleres o parcelas demostrativas, a partir de las cuales se generan una serie de recomendaciones o propuestas técnicas más genéricas orientadas a grupos o a productores en general.

Por último, también dentro de las áreas de intervención fundamentales puede encontrarse el trabajo a nivel grupal o asociativo, señalado por 36 entrevistados, correspondientes al $90 \%$ de la muestra. A veces, esto refiere a "trabajar lo organizativo" o a dar "apoyo a organizaciones", mientras que en otras oportunidades se lo plantea como acompañamiento "a los procesos grupales". Como se mencionó anteriormente, este trabajo a nivel grupal o asociativo se orienta a fortalecer a los grupos de productores, para que puedan negociar con otros actores desde una posición de mayor solidez. A nivel más operativo, esto se busca a partir de la generación de espacios de discusión grupales orientados a construir consensos y líneas de acción conjuntas; de la capacitación de los productores sobre roles dentro de los grupos y organizaciones; y del apoyo a la obtención de personería jurídica. En definitiva, "se busca que se arme un grupo realmente, no que solamente se junten, sino que se formen, se consoliden como grupo primero, que tengan objetivos en común". 
Respecto de las áreas de intervención destacadas, se observa el trabajo con temáticas vinculadas con agua, presentes en 28 entrevistas (70\%) y el apoyo a la comercialización, en este caso en 23 entrevistas $(57,5 \%)$. El agua es un recurso fundamental tanto para la actividad agropecuaria como para el sustento de la vida humana. Por esto, no resulta extraño que constituya un área de acción destacada de los extensionistas dado que, como se plantea en las entrevistas, "[hay] muchos lugares con problemas de agua". Las acciones en esta área son varias, destacándose la gestión de proyectos orientados a la realización de perforaciones, la protección de vertientes, la construcción de represas comunitarias y la instalación de sistemas de distribución de agua a nivel local.

Respecto del trabajo en el ámbito de la comercialización, la mayor parte de las referencias de los entrevistados son genéricas, apuntado al trabajo en el área. Dentro de las acciones específicas se habla de la realización de estudios de mercado, de la capacitación en comercialización y marketing, de la coordinación de la producción y de la participación en remates. No obstante, tanto desde el INTA como desde la SAF, la opción más mencionada es el apoyo a ferias francas, mercados locales en los cuales los productores se conectan de manera directa con los consumidores. Aquí, resulta importante destacar que las ferias francas en Argentina tienen su máximo desarrollo en las provincias del noreste, especialmente en la de Misiones (GOLSBERG et al., 2010), recibiendo el mayor apoyo de parte de estas instituciones.

Finalmente, también deben mencionarse una serie de líneas de acción implementadas por los extensionistas rurales, pero cuya presencia cuantitativa en las entrevistas fue mucho menor. Dentro de ellas se incluye el apoyo a la incorporación de valor agregado a la producción primaria, propuesta indicada por 10 extensionistas; y el trabajo con problemáticas de tierra junto con la organización de eventos, como ferias ganaderas o de semillas, ambos referidos por 7 entrevistados cada uno.

\subsection{Dimensión social, actitudinal e interdisciplinaria de la concepción de extensión rural}

Tradicionalmente, en América Latina la ER ha sido y es una práctica asociada casi exclusivamente con técnicos y profesionales provenientes del ámbito de las ciencias agrarias (LANDINI y BIANQUI, 2014b). En Argentina esta tendencia se mantiene, aunque con diferencias entre las instituciones, observándose un perfil algo más interdisciplinario en la SAF (LANDINI, 2013b). De hecho, la muestra de este estudio está compuesta en un $90 \%$ por extensionistas provenientes del área técnica.

En contraste, los entrevistados mencionan de manera insistente en las entrevistas el carácter constitutivo de la dimensión social en las prácticas de ER que realizan. Uno de los extensionistas lo expresa de esta manera: "yo creo que nuestro trabajo [...] mucho es la parte social, mucho es la contención, hablar con la gente, hablar con los jóvenes, y después se va dando la parte técnica, lo que nosotros hacemos mucho es la parte social". Muchas veces, la referencia a 'lo social' es utilizada por el personal técnico para referirse al abordaje de problemáticas como agua, tierra, vivienda, salud, caminos o luz eléctrica, entre otras. Sin embargo, un análisis más amplio permite incluir dentro de la dimensión social de la ER el trabajo organizativo y de fortalecimiento grupal, la gestión de procesos participativos, la articulación interinstitucional, y las prácticas educativas asociadas al trabajo de los extensionistas. Así, en términos generales, se observa en los entrevistados una concepción integral del desarrollo rural, que no lo identifica con un simple aumento de la producción. Como explica un técnico, "muchas veces no mejora su calidad de vida duplicando la producción". Por el contrario, el desarrollo rural queda definido como una articulación entre lo productivo, el acceso a servicios y el desarrollo organizativoinstitucional: "obviamente que el desarrollo rural va más allá... es también desarrollar a quienes no tienen luz eléctrica, todavía no tiene agua..." 
Al mismo tiempo, los entrevistados no sólo reconocen a 'lo social' como herramienta de la ER y como objetivo de su accionar, sino que también señalan la importancia de las características personales-actitudinales de los extensionistas para alcanzar buenos resultados en el trabajo. En concreto, los entrevistados hablan de la necesidad de ser una persona humilde, que sepa escuchar, que sea empática y tenga facilidad para generar relaciones de confianza, siempre en el marco de un vínculo horizontal, como destacaba Paulo Freire (1973) en su enfoque dialógico de ER. Un entrevistado lo señala así: “y con respecto a la actitud [del extensionista] es una relación de igual a igual con el otro, escuchar, tener una actitud humilde".

En este contexto, 17 entrevistados señalaron, ya sea de manera directa o indirecta, la limitación de la formación recibida para llevar adelante las tareas que corresponden a su función como extensionistas. Dado que la amplia mayoría de ellos poseen formación en ciencias agrarias, la primera limitación se relaciona con la falta de conocimientos en el ámbito social, organizativo y de metodologías de ER: "nosotros somos ingenieros agrónomos, lamentablemente no tenemos la formación social [...]. Nosotros estamos preparados para otras cosas". A la vez, también hay entrevistados que destacan la limitación de su propia formación técnica, ya que ésta está orientada fundamentalmente a la gran producción y a la búsqueda de maximización de la rentabilidad económica, cuando la agricultura familiar se encuentra organizada según una dotación de capital y una lógica diferente: "la facultad está orientada a generar agrónomos para las grandes producciones, para los commodities, para este sector no". Finalmente, también está la cuestión de la formación específica en ER, ya que en las carreras de agronomía y veterinaria, la ER suele ser una materia $u$ optativa o que tiene una posición periférica en los programas de estudio.

En este contexto, en el cual los extensionistas reconocen que la ER requiere de conocimientos y competencias que exceden a su propia formación, 25 entrevistados (es decir, el 62,5\%) destacaron la importancia de que el trabajo de ER sea sostenido por equipos interdisciplinarios, coincidiendo en esto con la propuesta de diferentes autores (CARBALLO, 2002, ORTIZ et al., 2011; PREISSING et al., 2014). Uno de los extensionistas aclara esto: “10 ojos ven más que 2 , si no, es muy limitado el trabajo que podés hacer solo. Necesitás de otras disciplinas". Efectivamente, tanto el trabajo en equipo como la interdisciplina permiten ampliar la comprensión de los fenómenos sociales complejos con los que deben trabajar los extensionistas (véase LANDINI et al., 2014). Cuando las concepciones de ER, desarrollo e innovación se amplían, también se multiplican las miradas que tienen que aportar a la comprensión del escenario de trabajo: "eso es lo que enriquece un trabajo, tener diferentes miradas sobre un mismo problema y tratar de trabajar de esa manera, en forma conjunta". Así, la necesidad de interdisciplina aparece como la respuesta a la ampliación del ámbito de intervención de los extensionistas y al aumento de la profundidad y complejidad de la lectura que deben hacer de los territorios en los que trabajan.

\section{Reflexiones finales y conclusiones}

En este trabajo se propuso describir el enfoque y las prácticas de ER de los extensionistas del noreste argentino, relacionarlas con las propuestas académicas e institucionales actuales de ER y extraer aprendizajes para fortalecer el impacto de las acciones. A continuación se presentan un conjunto de reflexiones, que terminan en una síntesis final.

En cuanto a los objetivos del trabajo de ER planteados por los entrevistados, se destaca una interesante articulación entre una orientación técnico-productiva, más tradicional, y un importante énfasis puesto en el fortalecimiento organizativo e institucional de los productores, esto último en línea con recomendaciones derivadas de diferentes estudios (SWANSON, 2010; ORTIZ et al., 2011). En este contexto, llama la atención la fuerte carga ético-política 
que asocian numerosos entrevistados a la idea de organización de los productores, en tanto estrategia para fortalecer o empoderar al sector en el contexto de unas relaciones sociales desiguales que marginan a los pequeños agricultores. Esta perspectiva, presente en una parte importante de los extensionistas, vuelve a aparecer cuando se plantean discusiones en torno a los modelos de desarrollo y de sociedad que subyacen a diferentes prácticas de extensión y modelos tecnológicos, todo lo que genera lecturas en torno al trabajo de ER en clave de relaciones de poder. Así, para numerosos entrevistados, la ER no aparece ni como una práctica tecnocrática ni como una simple intervención técnica, sino como una práctica política (en un sentido amplio, no partidario, vinculado con la definición de los fines hacia donde orientar la sociedad).

Este enfoque, que no suele caracterizar ni a la literatura generada por organismos internacionales como la FAO ni a las propuestas académicas referidas a ER desarrolladas en los países centrales, particularmente anglosajones, sí se articula fuertemente con la tradición latinoamericana. En concreto, retoma los postulados de la epistemología histórico-crítica presentes en la ER dialógica propuesta por Paulo Freire (1973), quien analiza la articulación saberpoder en las dinámicas de conocimiento de la ER, colocándolas en el contexto más amplio de las relaciones sociales de opresión. A la vez, esta mirada de la extensión como práctica política, también se apoya en las críticas a los postulados de la revolución verde contenidos en las propuestas de extensión que hacen foco en la agroecología (ALEMANY y SEVILLA GUZMÁN, 2007), lo que es recogido a nivel latinoamericano especialmente por la Política Nacional de Assistência Técnica e Extensão Rural (PNATER) de Brasil (MINISTÉRIO DO DESENVOLVIMENTO AGRÁRIO, 2004).

En cuando a los ejes del enfoque de ER surgidos de las entrevistas con los extensionistas, el énfasis dado a la orientación a la demanda en contraste con la idea más profunda de participación, si bien no resulta llamativa (ya que parece ir en línea con la idea de participación a veces diluida que se presenta en la literatura generada por organismos internacionales), no por eso deja de generar cierta preocupación. En efecto, numerosos problemas identificados por los extensionistas en su práctica, como la falta de compromiso, implicación y continuidad de los productores en relación a diferentes iniciativas y proyectos, parecen relacionarse con la falta de espacios de participación real en su diseño (SARAIVA y CALLOU, 2009; RIVAS et al., 2010). Así, es posible que la disponibilidad de la alterativa menos problemática de 'responder a la demanda', leída por los propios extensionistas como 'participación', limite los esfuerzos y las acciones orientadas a generar formas de participación más sustanciales. En este sentido, cabe una reflexión sincera por parte de los extensionistas de hasta qué punto la idea de simplemente responder a la demanda es suficiente para generar procesos que tengan impacto y continuidad a largo plazo, e incluso si esta alternativa no estará limitando los esfuerzos para generar dinámicas participativas más profundas, de mucha más difícil implementación.

Por otra parte, y hasta cierto punto, también se observa una coincidencia en torno a las justificaciones relacionadas con la preferencia por abordajes grupales en el trabajo de ER y con las acciones orientadas a la articulación interinstitucional. En ambos casos se tienden a dar explicaciones extrínsecas, que no destacan la utilidad o los beneficios de dichos abordajes en sí mismos. Respecto del trabajo grupal, se destaca la falta de recursos materiales y humanos para abordajes individuales, y en el caso de la articulación interinstitucional se enfatiza la necesidad de aprovechar recursos provenientes de otras instituciones. Así, se llega a la conclusión de que estas acciones se apoyan en explicaciones de carácter pragmático, no relacionadas con sus beneficios o su valor intrínseco.

En el caso del formato grupal de trabajo, si bien en otro contexto se ha destacado el valor de las organizaciones de productores en términos de capital social, lo que tiende a quedar invisibilizado es la potencialidad de los espacios grupales 
como herramientas para el intercambio de conocimientos y la facilitación de aprendizajes a nivel horizontal, lo que resulta fundamental si se piensa en desarrollar capacidades de innovación más que en transferir tecnologías. En este sentido, resulta de interés implementar acciones para que los extensionistas tomen conciencia de estas potencialidades y puedan maximizar los aprendizajes que pueden generarse en el contexto de los grupos.

Por su parte, lo que tiende a quedar invisibilizado en el caso de la articulación interinstitucional y de la implementación de una ER territorial (en este caso de manera algo más matizada), es el valor de la coordinación de políticas a nivel local y la construcción de lineamientos de desarrollo compartidos. En cierto sentido, parecería que en su operativización, el abordaje territorial propuesto por el INTA perdiera parte de su fuerza, quedando preso de las necesidades más cotidianas de articulación operativa.

Siguiendo con los ejes a partir de los cuales se estructura el trabajo de ER, en el desarrollo de los resultados también llamó la atención el escaso interés de la temática de género en los entrevistados, especialmente en los hombres. Sin dudas, para arribar a resultados definitivos resultaría necesario implementar una estrategia de investigación más directa. No obstante, los hallazgos de este estudio invitan a reflexionar sobre el espacio que dan las instituciones de ER al género en sus capacitaciones y en sus proyectos, más allá de lo que sería la simple conformación de grupos de trabajo específicamente para mujeres.

Focalizando ahora en las áreas de acción de los extensionistas, entre los resultados se mencionó la importancia que tenía la formulación de proyectos en el contexto del trabajo de ER. En contraste, se observaron escasas referencias a la evaluación de dichos proyectos. Tradicionalmente, la idea de evaluación ha sido asociada a la de control (NIREMBERG et al., 2000). En este sentido, evaluar los proyectos implementados se operativiza a partir de la presentación de planillas y comprobantes. Así, lo que termina quedando de lado es la potencialidad de la evaluación como aprendizaje, al reflexionar sobre lo hecho identificando aciertos, errores y estrategias superadoras. En consecuencia, en el contexto del trabajo de ER, resulta recomendable aprovechar la potencialidad de la evaluación para aprender, generando institucionalmente espacios en los que sea posible sistematizar experiencias, generar aprendizajes y compartirlos, desarrollando así organizaciones con alta capacidad de innovación.

Respecto de las áreas de intervención de los extensionistas, la investigación permitió identificar tres fundamentales, la articulación interinstitucional, el trabajo grupal o asociativo, y la asistencia técnica, a las cuales deben sumarse el abordaje de la problemática del agua y el apoyo a la comercialización, ambas con menor presencia cuantitativa. Este panorama muestra una ER que mantiene el interés productivo tradicional, pero al que se suma la articulación interinstitucional (como expresión del abordaje de ER territorial propuesto por el INTA), y el fortalecimiento organizacional, relacionado con la formación de capital social en el contexto de la agricultura familiar, en línea con la interpretación en términos de relaciones de poder de la posición del sector en el contexto de la economía. A la vez, a esto se incorporan problemáticas sentidas por los productores, como el acceso al agua y la comercialización.

Atendiendo a este contexto complejo de práctica, los extensionistas entrevistados destacaron, tanto de manera directa como indirecta, la necesidad de articular lo social con lo productivo en la práctica de la extensión, a diferentes niveles. En primer lugar, se observa la necesidad de incorporar profesionales formados tanto en disciplinas técnicas como en ciencias sociales, con el fin de generar una visión amplia de la realidad sobre la que se interviene, así como sobre sus determinantes. De esto se sigue la expectativa de un abordaje interdisciplinario de la labor de ER, lo que termina contrastando con el bajo porcentaje de extensionistas provenientes del ámbito social, particularmente en el INTA (LANDINI, 2013b). Ante esta situación cabe 
preguntarse por las alternativas para generar equipos de trabajo interdisciplinarios en los diferentes territorios $\mathrm{o}$, al menos, para incorporar asesoramiento social en los equipos formados exclusivamente por personal técnico.

Por su parte, la articulación entre la dimensión social y la técnica en el contexto de la ER no sólo refiere a las disciplinas implicadas, sino también al contenido del trabajo. En efecto, la descripción de objetivos, actividades y áreas de intervención incorporó tanto aspectos productivos como sociales, incluyendo acceso a agua y tierra, organización social y articulación interinstitucional, entre otros. De esta manera, se observa la emergencia de una concepción integral de desarrollo rural, que trasciende ampliamente la dimensión productiva.

Ante esta situación, la mayor parte de los extensionistas destacaron las limitaciones de su formación para enfrentar la diversidad y complejidad de su práctica. Esto lleva a poner en un lugar de reflexión destacado la formación de quienes trabajan en extensión (RYAN et al., 2012; BOCCHICCHIO, 2013; LANDINI, 2013c). En concreto, icuáles son las estrategias que utilizan las instituciones de ER para formar a sus extensionistas? ¿Cómo se relacionan estas estrategias con la multiplicidad de problemáticas que éstos deben enfrentar en su práctica? Por último, y no por esto menos importante, ¿cómo se están aprovechando y potenciando las posibilidades de aprendizaje relacionadas con la reflexión sobre la práctica, la sistematización de experiencias, y el intercambio horizontal entre extensionistas? Si bien no se ha recabado información específica en este proyecto, resulta claro que existen múltiples oportunidades para mejorar las estrategias de formación implementadas por las instituciones, lo que se conoce como formación en servicio.

En resumen, se observa un enfoque y una práctica de ER difícil de reducir o de categorizar dentro de un abordaje único, por múltiples razones, las que incluyen tanto la existencia de prácticas diversas y de miradas contrapuestas entre los propios extensionistas como la presencia de justificaciones para dichas prácticas que no son necesariamente consistentes con los modelos que las proponen. De cualquier manera, y a grandes rasgos, se reconoce en las prácticas descriptas la influencia del abordaje territorial propuesto por el INTA. No obstante, quedan dudas respecto de: (1) el valor intrínseco dado al abordaje grupal y al trabajo interinstitucional, (2) la importancia dada a formas sustanciales de participación, en contraste con la simple 'orientación a la demanda', y (3) el rol asignado al enfoque de género y a la evaluación de los proyectos implementados. A la vez, también se identificaron dos elementos que en cierta medida parecen trascender lo que usualmente se piensa como parte de la ER como son: (1) el énfasis en la dimensión éticopolítica dado por los extensionistas a su práctica, y (2) la consideración de lo social no sólo como herramienta sino también como objetivo de las acciones de extensión. Por último, también cabe destacar que la tipología de enfoques de ER propuesta en la introducción, que incluyó los enfoques difusionista, dialógico, territorial y de sistemas de innovación, si bien aportó a la organización de los resultados, también se vio desbordada por la diversidad de concepciones y prácticas identificadas en esta investigación.

\section{Referencias}

ACUNZO, M. et al. Communication for rural development. Roma: FAO, 2014.

ADGABA, N. et al. Socio-economic analysis of beekeeping and determinants of box hive technology adoption in the Kingdom of Saudi Arabia. Journal of Animal \& Plant Sciences, v. 24, n. 6, p. 1876-1884, 2014.

ALEMANY, C. y SEVILLA GUZMÁN, E. ¿Vuelve la extensión rural? Reflexiones y propuestas agroecológicas vinculadas con el retorno y fortalecimiento de la extensión rural en América Latina. Realidad Económica, n. 227, p. 52-74, 2007.

ALMEIDA, S., OLIVEIRA, M. y XAVIER, J. A descentralização da política nacional de ATER: uma experiência nos assentamentos de reforma agrária no noroeste mineiro-Brasil. Sociedade \& Natureza, v. 22, n. 3, p. 551-560, 2010. 
ARDILA, J. Extensión rural para el desarrollo de la agricultura y la seguridad alimentaria: Aspectos conceptuales, situación y una visión de futuro. San José, Costa Rica: IICA, 2010.

BOCCHICCHIO, A. Perfil profesional en el área de extensión y desarrollo rural. En: FEITO, M., BATTISTA, S., CORIA, L. y LANDINI, F. (Eds.). Contribuciones internacionales al desarrollo local y rural: Hacia una agenda de investigación. San Justo, Argentina: Universidad Nacional de La Matanza, 2013, p. 184-188.

CARBALLO, C. Extensión y transferencia de tecnología en el sector agrario argentino. Buenos Aires: Universidad de Buenos Aires, 2002.

CHRISTOPLOS, I. Cómo movilizar el potencial de la extensión agraria y rural. Roma: FAO, 2010.

DE LELIS, D., COELHO, F. y DIAS, M. A necessidade das intervenções: Extensão rural como serviço ou como direito? Interações, v. 13, n. 1, 69-80, 2012.

FERRO, S. Estudio comparativo regional de asistencia técnica y extensión rural con perspectiva de género. Programa Regional de Género de la Reunión Especializada de la Agricultura Familiar del MERCOSUR, 2014. Disponible en: <http://redaf.org.ar/wp-content/uploads/2014/12/ ferro_asistencia\% 20 _tecnica_y_xtension_rural_ genero2014.pdf>. Acceso en: 7 de julio de 2015 .

FORO GLOBAL PARA LOS SERVICIOS DE ASESORÍA RURAL. Marco estratégico a largo plazo (2011-2016). Lindau, Suiza, 2012. Disponible en: <http://www.g-fras.org/en/knowledge/documents/ category/16-strategic-and-management-documents. html? download =128:gfras-marco-estrategico $>$. Acceso en: 8 de marzo de 2015.

FREIRE, P. ¿Extensión o comunicación? La concientización en el medio rural. Buenos Aires: Siglo XXI, 1973.

GOLSBERG, C. et al. Las ferias de la agricultura familiar en la Argentina. Buenos Aires: INTA, 2010.

INSTITUTO NACIONAL DE TECNOLOGÍA AGROPECUARIA. El INTA que queremos: Plan Estratégico Institucional 2005 - 2015. Buenos Aires: INTA, 2004.

LANDINI, F. Problemas en la extensión rural paraguaya: modelos de extensión en la encrucijada. Cuadernos de Desarrollo Rural, v. 9, n. 69, p. 127-149, 2012.

. Problemas enfrentados por los extensionistas rurales argentinos en el ejercicio de su labor desde su propia perspectiva. Revista de Economia e Sociologia Rural, v. 51, n. sup1, p. s079-s100, 2013a.
. Perfil de los extensionistas rurales argentinos del sistema público. Mundo Agrario, v. 14, n. 27, 2013 b. Disponible en: < http://www.memoria.fahce.unlp.edu. ar/art_revistas/pr.5947/pr.5947.pdf>. Acceso en: 12 de julio de 2015.

. Necesidades formativas de los extensionistas rurales paraguayos desde la perspectiva de su función, sus problemas y sus intereses. Trabajo y Sociedad, n. 20, 149-160, 2013c.

Concepción de 'extensión rural' de los extensionistas rurales argentinos que trabajan en el sistema público nacional con pequeños productores. Cuadernos de Desarrollo Rural, v. 12, n. 75, p. 35-53, p. 2015 a.

. Análisis de la extensión rural salvadoreña a partir de la mirada de sus extensionistas. Interações, v. 16, n. 2 , p. $455-464,2015$ b.

Problemas enfrentados por extensionistas rurais brasileiros e sua relação com suas concepções de extensão rural. Ciência Rural, v. 45, n. 2, p. 371-377, 2015c.

Different Argentine rural extensionists' mindsets and their practical implications. The Journal of Agricultural Education and Extension, v. 21, n. 3, p. 219234, 2015d.

., BIANQUI, V. y CRESPI, M. Evaluación de las creencias sobre extensión rural de los extensionistas paraguayos. Psiencia, v. 5, n. 1, p. 3-14, 2013.

., LEEUWIS, C., LONG, N. y MURTAGH, S. Towards a psychology of rural development processes and interventions. Journal of Community and Applied Social Psychology, v. 24, n. 6, p. 534-546, 2014.

. y BIANQUI, V. La extensión rural en Ecuador desde el punto de vista de sus extensionistas. Revista de la Facultad de Agronomía (Universidad de Zulia), v. 31, n. 3, p. $433-454,2014$ a.

. y BIANQUI, V. Socio-demographic profile of different samples of Latin American rural extensionists. Ciência Rural, v. 44, n. 3, p. 575-581, 2014 b.

LEEUWIS, C. Communication for rural innovation: Rethinking agricultural extension. Oxford: Blackwell Science, 2004.

. y PYBURN, R. (Eds.). Wheelbarrows full of frogs: Social learning in rural resource management. Assen, Holanda: Van Gorcum, 2002.

- y AARTS, N. Rethinking communication in innovation processes: creating space for change in complex systems. Journal of Agricultural Education and Extension, v. 17, n. 1, p. 21-36, 2011. 
LONG, N. Sociología del desarrollo: una perspectiva centrada en el actor. México: Centro de Investigaciones y Estudios Superiores en Antropología Social, 2007.

MACHADO, J., DE HEGEDÜS, P. y SILVEIRA, L. Estilos de relacionamento entre extensionistas e produtores. Ciência Rural, v. 36, n. 2, 2006.

MANZANA, N. et al. Optimal feeding systems for small-scale dairy herds in the North West Province, South Africa. Journal of the South African Veterinary Association, v. 85, n. 1, 2014. Disponible en: < http:// www.ncbi.nlm.nih.gov/pubmed/25026955>. Acceso en: 12 de julio de 2015.

MINISTÉRIO DO DESENVOLVIMENTO AGRÁRIO. Política Nacional de Assistência Técnica e Extensão Rural, 2004. Disponible en: <http://www.mda.gov.br/ sitemda/sites/sitemda/files/user_arquivos_64/Pnater. pdf $>$. Acceso en: 15 de diciembre de 2015.

MIRANDA, T. et al. La innovación y la transferencia de tecnologías en la Estación Experimental "Indio Hatuey": 50 años propiciando el desarrollo del sector rural cubano (Parte I). Pastos y Forrajes, v. 34, n. 4, p. 393412, 2011.

MONTERO, M. Introducción a la psicología comunitaria: Desarrollo, conceptos y procesos. Guadalajara: Universidad de Guadalajara, 2004.

NEDERLOF, E., WENNINK, B. y HEEMSKERK, W. Access to agricultural services. Amsterdam: Royal Tropical Institute, 2010.

NIREMBERG, O., BRAWERMAN, J. y RUIZ, V. Evaluar para la transformación. Innovaciones en la evaluación de programas y proyectos sociales. Buenos Aires: Paidós, 2000.

OLORUNFEMI, O. et al. Ensuring improved livelihood opportunities for resource-poor maize farmers through the dissemination of striga control methods in Kwara State, Nigeria. Albanian Journal of Agricultural Sciences, v. 13, n. 2, p. 80-88, 2014.

ORTIZ, R. Evolución de los servicios de extensión en Nicaragua. Roma: FAO, 2009.

. et al. Buenas prácticas en el manejo de extensión en América Central. Roma: FAO, 2011.

PAVÓN, M. Extensionismo en Cuba: Estudios de caso. Cultivos Tropicales, v. 35, n. 1, p. 5-10, 2014.

PEIXOTO, A. y PEREIRA, R. Discurso versus ação no comportamento ambientalmente responsável. Revista de Gestão Ambiental e Sustentabilidade, v. 2, n. 2, p. 71-113, 2013.
PÉREZ, M. y CLAVIJO, N. Experiencias y enfoques de procesos participativos de innovación en agricultura. El caso de la corporación PBA en Colombia. Roma: FAO, 2012.

PREISSING, J., ARDILA, S., BUITRÓN, J. y FERNÁNDEZ, C. Nuevas inversiones en extensión para la agricultura familiar. En SALCEDO S. y GUZMÁN L. (Eds.). Agricultura familiar en América Latina y el Caribe: Recomendaciones de política. Santiago de Chile: FAO, 2014, p. 215-232.

QAMAR, K. Introducing demand-driven extension approach in a traditional region: a case study from Pakistan. Roma: FAO, 2011.

RENDÓN, R. et al. Los procesos de extensión rural en México. Revista Mexicana de Ciencias Agrícolas, v. 6, n. 1, p. 151-161, 2015.

RINGUELET,R. Los estudios sociales dely para el desarrollo rural. Mundo Agrario, v. 10, n. 20, 2010. Disponible en: $<$ http://www.scielo.org.ar/pdf/magr/v10n20/v10n20a18. pdf $>$. Acceso en: 17 de octubre de 2015.

RIVAS, A., AVENDAÑO, P. y QUINTERO, H. Updating peasant competencies to mitigate poverty in the Chorti community, Copan (Honduras). Agronomía Colombiana, v. 28, n. 3, p. 567-575, 2010.

ROGERS, E. Diffusion of innovations. Nueva York: Free Press, 1962.

RYAN, J. et al. Training and capacity building: an essential strategy for development at an international research center. Sustainable Agriculture Research, v. 1, n. 2, p. 57-71, 2012.

SARAIVA, R. y CALLOU, A. Políticas públicas e estratégias de comunicação para o desenvolvimento local de comunidades pesqueiras de Pernambuco. Interações, v. 10, n. 1, p. 73-81, 2009.

SCHALLER, N. Extensión rural: ¿hacia dónde vamos?, ¿hacia dónde ir? El Colorado, Argentina: INTA, 2006.

SCHEJTMAN, A. y BERDEGUÉ, J. Desarrollo territorial rural. Santiago, Chile: Centro Latinoamericano para el Desarrollo Rural, 2004.

SOUZA, I. y GOMES, A. Folkcomunicaçao e extensão rural brasileira: as estratégias de comunicação rural para o desenvolvimento local. Razón y Palabra, n. 60, 2008. Disponible en: <http://www.razonypalabra.org. $\mathrm{mx} /$ anteriores/n60/souza_gomes.htm $>$. Acceso en: 16 de octubre de 2015.

SULAIMAN, R. y DAVIS, K. The "New Extensionist": Roles, strategies, and capacities to strengthen extension and advisory services. Lindau, Suiza: Global Forum for Rural Advisory Services, 2012. 
SWANSON, B. Estudio mundial sobre buenas prácticas de los servicios de extensión y asesoramiento agropecuarios en el mundo. Roma: FAO, 2010.

TAYLOR, S. y BODGAN, R. Introducción a los métodos cualitativos de investigación. La búsqueda de significados. Barcelona: Paidós, 1994.

TOMMASINO, H., GONZÁLEZ, M., GUEDES, E. y PRIETO, M. Extensión crítica: los aportes de Paulo Freire. En: TOMMASINO H. y DE HEGEDÜS, P. (Eds.). Extensión: reflexiones para la intervención en el medio urbano y rural. Montevideo: Universidad de la República, 2006, p. 121-135.

TORT, M. Enfoques de la extensión rural. En nuestro agro: ¿Evolución, complementación u oposición. En BALSA, J., MATEO, G. y OSPITAL, M. (Comp.). Pasado $y$ presente en el agro argentino. Buenos Aires: Lumiere, 2008, p. 428-450.
TRIGO, E., MATEO, N. y FALCONI, C. Agricultural innovation in Latin America and the Caribbean: Institutional scenarios and mechanisms (Technical Note No. IDB-TN-528). Banco Interamericano de Desarrollo, 2013. Disponible en: <http://www.g-fras.org/media/ kunena/attachments/52/IDBDOCS-37938256-v4Agricultural_Innovation_in_LAC.pdf >. Acceso en: 19 de octubre de 2014.

TURIJÁN, T. et al. Manejo tradicional e innovación tecnológica en cultivo de maíz en San José Chiapa, Puebla. Revista Mexicana de Ciencias Agrícolas, v. 3, n. 6, p. 1085-1100, 2012.

ZUIN, L., ZUIN, P. y MANRIQUE, M. A comunicação dialógica como fator determinante para os processos de ensino-aprendizagem que ocorrem na capacitação rural: um estudo de caso em um órgão público de extensão localizado no interior do Estado de São Paulo. Ciência Rural, v. 41, n. 5, p. 917-923, 2011. 